\title{
AMS -A powerful tool for probing nucleosynthesis via long-lived radionuclides
}

\author{
A. Wallner ${ }^{1, a}$, R. Golser ${ }^{1}$, W. Kutschera ${ }^{1}$, A. Priller $^{1}$, P. Steier ${ }^{1}$, and C. Vockenhuber ${ }^{1,2}$ \\ 1 VERA-Laboratory, Institut für Isotopenforschung und Kernphysik, Universität Wien, Währinger Strasse 17, A-1090 Wien, \\ Austria \\ 2 TRIUMF Laboratory, 4004 Wesbrook Mall, Vancouver, BC, V6T 2A3, Canada
}

Received: 3 July 2005 /

Published online: 23 March 2006 - (C) Società Italiana di Fisica / Springer-Verlag 2006

\begin{abstract}
Well-established data on production-rates of long-lived radionuclides are important for the understanding and calculation of various nucleosynthesis processes. However, lack of information exists for a list of nuclides as pointed out by nuclear-data requests. In addition, the search for supernova (SN)-produced radionuclides will give an improved insight into explosive scenarios. Accelerator mass spectrometry (AMS) represents a technique, which is capable to quantify such long-lived radionuclides using mass spectrometric methods. The potential of AMS is presented here as a powerful tool for probing nucleosynthesis. Applications of AMS are exemplified for a few specific cases: the detection of extraterrestrial radioactivity on Earth in terrestrial archives as a signature of nearby SN explosions, and the measurement of cross-sections, as an important ingredient for stellar as well as nuclear model calculations.
\end{abstract}

PACS. 07.75. $+\mathrm{h}$ Mass spectrometers $-26.20 .+\mathrm{f}$ Hydrostatic stellar nucleosynthesis $-26.30 .+\mathrm{k}$ Nucleosynthesis in novae, supernovae and other explosive environments - 26.35.+c Big Bang nucleosynthesis

\section{Introduction}

Nuclear astrophysics continues to be a rich and exciting research field. Pushed by the public interest, nuclear astrophysics is called to provide answers to various outstanding questions. In order to meet these inquiries, astrophysical research is a growing field [1]. Within this astrophysical context, nuclear-data activities are one basic instrument to help to answer such questions [2,3]. Nuclear data are needed for a better understanding of spectacular events such as the Big-Bang, star evolution and supernova (SN) explosions, but also to gain insight into the formation of our solar system. Nucleosynthesis gives us one of the very important fingerprints of nature, the elemental abundances. In order to understand this signature, the physics of nuclear reactions have to be well understood. Network calculations of element production in various scenarios have shown a great progress in the last years and they allow for reproducing a lot of the abundance distribution of the elements. However, we are still at a very early stage of understanding the physics leading to the well-known isotopic pattern of our Solar System. The success of astrophysical models depends strongly on the accuracy of nuclear data. These ingredients have to be provided from experimental investigations. One essential key to this request is the precise knowledge of reaction and production

\footnotetext{
a e-mail: anton.wallner@univie.ac.at
}

cross-sections. Such experimental input helps to verify nuclear model calculations and gives us a deeper understanding of the nuclear forces responsible for the nuclear interactions. This huge nuclear-data need pushes the activities of experimental facilities to investigate astrophysical questionnaires. Here we want to discuss the technique of accelerator mass spectrometry (AMS) as a valuable tool for probing nucleosynthesis. In the following sections the potential of AMS for quantifying long-lived radionuclides will be highlighted.

\section{Accelerator mass spectrometry at the Vienna Environmental Research Accelerator facility}

Well-established data on production-rates of long-lived radionuclides are important for the various nucleosynthesis processes and they are highly desired. Lack of information on cross-section data exists for a number of nuclides as pointed out by nuclear-data requests. Especially longlived radionuclides have often been inaccessible to decay counting techniques, e.g. because of low activity or an unfavorable decay scheme. In such cases the technique of AMS may provide valuable contributions to our understanding of celestial evolution and nucleosynthesis. 


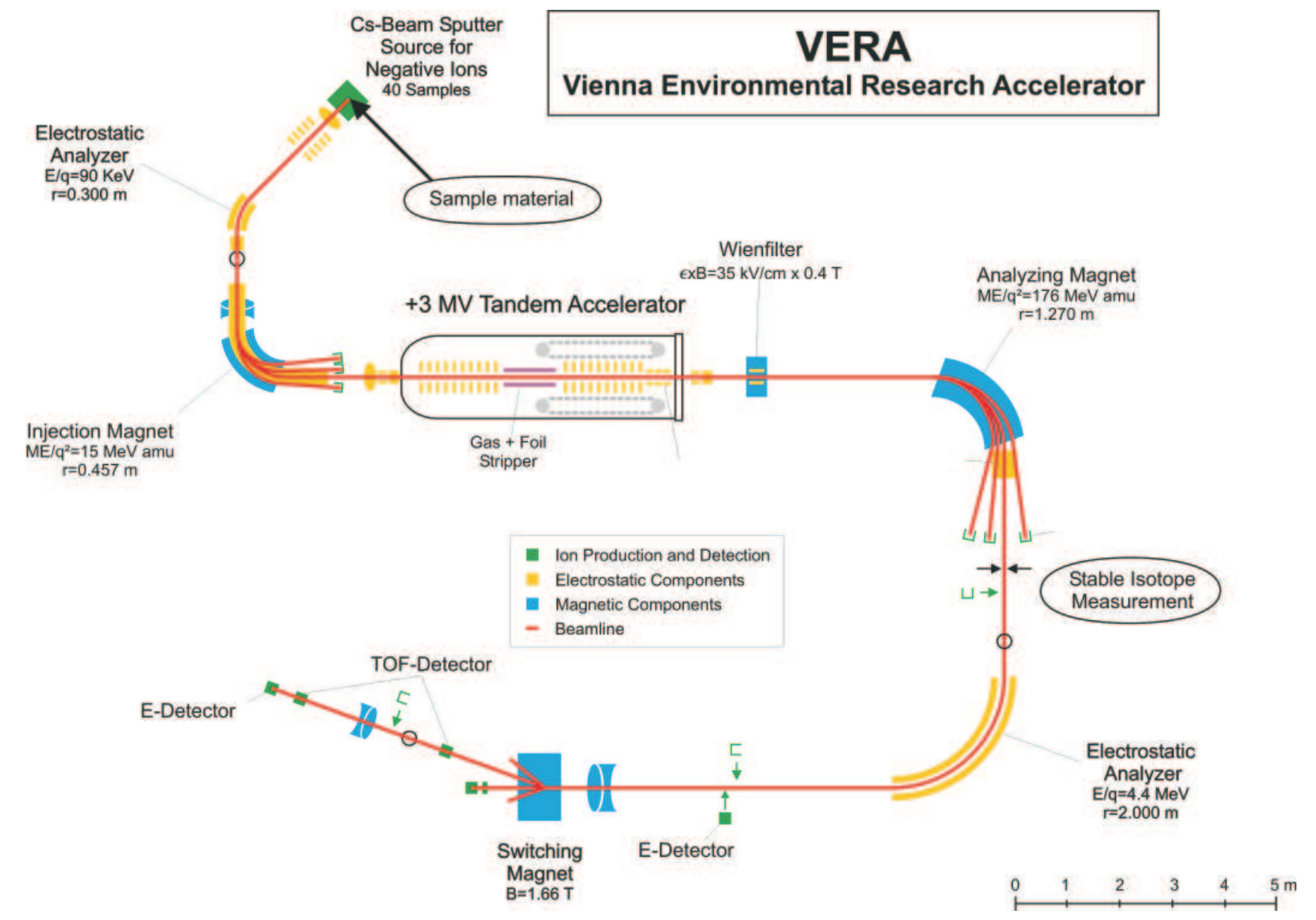

Fig. 1. Schematic layout of the VERA facility: Stable ions are measured with Faraday cups positioned after the injection magnet (low-energy section, negative ions) and after the analyzing magnet (high-energy section, positive ions). The rare isotope count-rates are obtained using energy-sensitive particle detectors. The detection system of the heavy-ion beamline (which is also used for ${ }^{41} \mathrm{Ca}$-detection) consists of the time-of-flight (TOF) detectors and an energy (E) detector.

Accelerator mass spectrometry (AMS) represents an analytical method for the detection of various nuclides through direct atom-counting. This mass spectrometric technique offers the potential for quantifying isotope abundances down to levels of $10^{-15}$ and below. AMS is applied for the detection of radionuclides with half-lives between a few years and up to hundred million years. In combination with very low sample masses needed, AMS offers a tremendously higher sensitivity compared to the decay counting method, which is a consequence of the long half-life of those radionuclides.

In the following the method of AMS is summarized with focus on a typical AMS facility as represented by the Vienna Environmental Research Accelerator (VERA). VERA is based on a 3-MV pelletron tandem. It represents a state-of-the-art AMS facility (fig. 1), which provides the ability for quantifying nuclides over the whole mass range $[4,5,6]$. Some of the measured radioisotopes are, e.g. $,{ }^{10} \mathrm{Be},{ }^{14} \mathrm{C},{ }^{26} \mathrm{Al},{ }^{36} \mathrm{Cl},{ }^{41} \mathrm{Ca},{ }^{129} \mathrm{I},{ }^{182} \mathrm{Hf},{ }^{236} \mathrm{U}$ and ${ }^{244} \mathrm{Pu}$. Since it offers highest sensitivity, AMS at VERA is predestinated for the measurement of minute concentrations of such isotopes. At VERA, AMS is used for quan- tifying long-lived radionuclides within a wide range of applications - from archaeology via climate research to astrophysics (for details see, e.g., $[7,8]$ ).

AMS uses negative ion sputter sources: Solid sample material is inserted into an ion source. At VERA a samplewheel containing positions for 40 samples can be loaded into the ion source. The sample material is sputtered using a Cs beam focused onto the sample. In most cases the sample material has to be converted for the AMS measurement into a suitable chemical form. AMS itself represents a "sample-destructive" technique. Typical samples masses are a few $\mathrm{mg}$ of material. The range of isotope ratios measured with AMS is of the order of $10^{-10}$ to $10^{-15}$. It is also this dynamic range, which makes AMS a flexible and attractive technique. The sensitivity of AMS is exemplified using the following typical values: Assume $10 \mathrm{mg}$ sample material (e.g., Ca) is available for a measurement (which will give several sputter targets for the AMS measurement). If we combine a typical isotope ratio of $10^{-12}$ (or $10^{-14}$ ) and the sample mass, we obtain a total number of rare isotopes of about $10^{8}\left(10^{6}\right)$ atoms. With an assumed half-life of 100000 years $\left(\lambda=2 \cdot 10^{-13} \mathrm{~s}^{-1}\right)$, these numbers 
correspond to activities of $20 \mu \mathrm{Bq}$ (or $0.2 \mu \mathrm{Bq}$ ), far below any chance to be quantified by activity measurements.

In AMS the typical measurement procedure is the following: In a cesium sputter source, negatively charged ions are produced and pre-accelerated before they pass a lowenergy mass spectrometer, which analyzes a specific mass. The ions are further injected into a tandem accelerator (at VERA we use up to $+3.5 \mathrm{MV}$ terminal voltage). Any molecules that might contribute to a molecular interference are completely destroyed in the terminal stripper of the accelerator. Due to this stripping process, only atomic, mostly positively charged ions leave the tandem accelerator. A specific charge state is selected by a second, highenergy $90^{\circ}$ analyzing magnet for further transport to the detector. At VERA using the 3-MV tandem, the particles have energies between 10 and $25 \mathrm{MeV}$. Stable isotopes will be measured as currents using Faraday cups while the radionuclides are counted directly with a particle detector. Rejection of isotopic interferences can be achieved with additional filters, like a Wien-filter, an electrostatic analyzer, and a time-of-flight system. Further reduction of any isobaric and isotopic interference is also achieved by means of specific energy-loss techniques, used either in front of the detection system or as a part of the particle detector (see also sect. 4, Ca-measurements with VERA). A final particle detector delivers the energy of the incoming ions while they are fully stopped. The detection beam-line at VERA is described in [6]; typical parameters for the various nuclides measured at VERA are listed in $[5,9]$.

AMS is a mass spectrometric technique. Basically, it measures isotope count rates for different isotopes. To this end, sequentially, stable ion currents are measured with Faraday cups, positioned at the low-energy and highenergy sides of the AMS beamline (fig. 1). These current measurements are sandwiched by counting the rare isotope with the particle detector. With this raw data, i.e. countrate and particle current, an isotope ratio is calculated. However, possible long-term drifts of the particle transmission along the beamline have to be monitored. For quality control, the transmission is regularly monitored by means of standards with well-known isotope ratios. Another ion may also mimic a true event in the detector. Contamination from chemistry or in the ion source may induce an additional "true" signal. In addition, isobaric interferences have to be controlled. Note, that impurities in the sample after chemical preparation are typically on the ppm-level, whereas the rare isotope content is another 6 to 9 orders of magnitude lower. In order to quantify or check the background level, blank samples are therefore measured, too.

The overall efficiency ( $i . e$. fraction of particles detected to that inserted into the ion source), which includes the efficiency for producing negative ions, stripping yield, transmission through the beam line and detector efficiency, depends strongly on the isotope under investigation. For carbon measurements up to several percent can be obtained at VERA but in other cases one has to deal with an overall efficiency as low as $10^{-5}[9]$.

\section{Applications of AMS related to astrophysics}

\subsection{Search for supernova-produced radionuclides on Earth}

Ellis, Fields and Schramm 1996 [10] and Fields [11] pointed out that there might be a chance for finding longlived radionuclides in terrestrial archives, which were originally produced in a supernova (SN). Several candidates had been identified by [10], among them ${ }^{26} \mathrm{Al},{ }^{53} \mathrm{Mn},{ }^{60} \mathrm{Fe}$, ${ }^{146} \mathrm{Sm},{ }^{182} \mathrm{Hf}$, and ${ }^{244} \mathrm{Pu}$ which are produced in sufficient amounts to be in principle detectable on Earth. Such radioisotopes, which have been ejected into the interstellar medium, may be picked up by our Solar System when it passes through such a region and those nuclides will finally become incorporated into terrestrial archives, like sediments or ice cores. SN-rates in our stellar vicinity, are expected to be rather common. In a rough estimation Fields [11] calculates a rate of the order of a few SN events per million years within a distance of 100 parsec (pc). From that follows, that proper radionuclides should have half-lives also in the million-year range, far too shortlived for surviving from the formation of the Solar System. Radionuclides with those half-lives are best measured using the technique of AMS. It is crucial to exclude contamination of these $\mathrm{SN}$-produced radionuclides from other natural sources (e.g., cosmic ray production, natural fission).

Indeed, with the use of AMS, a strong peak of ${ }^{60} \mathrm{Fe}$ $\left(T_{1 / 2}=1.5 \mathrm{Ma}\right)$ was found recently in a deep-sea manganese crust profile [12], which confirms the presumption of Ellis et al.. This unusual isotope signal is interpreted as live radioactivity deposited on Earth originating from a supernova explosion three million years ago, and at a distance of several tens of pc $[12,13]$. Hence, they are eagerly sought fingerprints of recent, explosive nucleosynthesis. However, this signal needs confirmation from other radionuclides. Possible other candidates are the r-process nuclides ${ }^{182} \mathrm{Hf}(8.9 \mathrm{Ma}),{ }^{244} \mathrm{Pu}$ (80.6 Ma), and ${ }^{247} \mathrm{Cm}(16 \mathrm{Ma})$. In some cases $\left(e . g .,{ }^{244} \mathrm{Pu}\right)$, man-made contributions have to be carefully evaluated.

The extremely low isotopic abundance of such radionuclides asks for a very sensitive detection method. Accelerator mass spectrometry (AMS) is currently the only technique capable to measure such ultra-low isotope ratios. At VERA, techniques for measuring the isotopes ${ }^{182} \mathrm{Hf}$ and ${ }^{244} \mathrm{Pu}$ have been developed $[34,14,15]$. Whereas the detection of SN-produced ${ }^{182} \mathrm{Hf}$ still suffers from interference from the stable isobar ${ }^{182} \mathrm{~W}$ and needs further technological developments, in the case of transuranium elements no stable isobar exists. Therefore, e.g. ${ }^{244} \mathrm{Pu}$ detection has to deal with the rejection of isotopic background only. The mass-selective low-energy injection system at VERA [16] and the dedicated low-background heavy-ion beamline in combination with the highly reproducible conditions make VERA a favorable system for measuring those isotopes. For the case of ${ }^{244} \mathrm{Pu}$ measurements, no isotopic interferences from other isotopes are found at VERA. This setup has also been proven to be very valuable for measuring the various other $\mathrm{Pu}$ isotopes in environmental samples [17]. In those cases, the man-made content of $\mathrm{Pu}$ is investigated 
which allows for example to reconstruct the neutron history of that environment. However, the isotopic signature of these other $\mathrm{Pu}$ isotopes $\left({ }^{239,240,241,242} \mathrm{Pu}\right)$ is a measure of the man-made ${ }^{244} \mathrm{Pu}$, too, because this information can be used to extrapolate the anthropogenic content of those $\mathrm{Pu}$ isotopes to the expected anthropogenic ${ }^{244} \mathrm{Pu}$ concentration in the sample material. Using the $14 \mathrm{MV}$ tandem accelerator in Munich, C. Wallner et al. found a ratio for ${ }^{244} \mathrm{Pu} /{ }^{239} \mathrm{Pu}$ of $10^{-3}[18]$. In a first measurement of a deepsea manganese crust they found one single count of ${ }^{244} \mathrm{Pu}$ in a background-free measurement. Since no ${ }^{239} \mathrm{Pu}$ was detected, no ${ }^{244} \mathrm{Pu}$ counts from anthropogenic sources are expected. A second, independent measurement to quantify ${ }^{244} \mathrm{Pu}$ in deep-sea sediment has been performed by $\mathrm{M}$. Paul et al. [19]. They also found one count of ${ }^{244} \mathrm{Pu}$ in their sample, however this signal is compatible with their expectations of anthropogenic origin using also the ${ }^{239} \mathrm{Pu}$ content of that sample.

In ref. [17] typical spectra from $\mathrm{Pu}$ measurements of environmental samples measured at VERA are shown. Best discrimination is obtained using the information of 2-dimensional spectra which comprise time-of-flight signals and energy signals. It is demonstrated that the highenergy mass separator is capable to suppress ions from neighboring masses completely which is important for detecting ${ }^{244} \mathrm{Pu}$. To summarize, using this setup, VERA has proven to be capable to quantify supernova-produced ${ }^{244} \mathrm{Pu}$ in terrestrial archives. If successful, the long half-life of ${ }^{244} \mathrm{Pu}(80 \mathrm{Ma})$ gives the possibility to trace the mean abundance of ${ }^{244} \mathrm{Pu}$ in the interstellar medium [18, 19,20], which may contain ejected material from several SN explosions.

\subsection{Measurement of production cross-sections}

The observation of live radionuclides provides direct evidence for ongoing nucleosynthesis. Radionuclides, like ${ }^{26} \mathrm{Al}[21]$ and ${ }^{44} \mathrm{Ti}$, can be traced in the sky as live radioactivity. Such mappings identify active areas in our Galaxy. The relevant stellar scenarios are an essential key for understanding celestial evolution. At VERA cross-section measurements have been performed, e.g. for the quantification of ${ }^{26} \mathrm{Al}[22,23]$. The background level for ${ }^{26} \mathrm{Al}$ detection at VERA is found to be at $6 \cdot 10^{-16}$. This extremely high sensitivity enables us also to apply AMS for the measurement of the ${ }^{25} \mathrm{Mg}(\mathrm{p}, \gamma){ }^{26} \mathrm{Al}$ strength functions at astrophysical interesting energies [24]. To this end, $\mathrm{Mg}$ targets have been irradiated with protons. After the irradiation the produced ${ }^{26} \mathrm{Al}$ is extracted for the successive AMS measurement [25,26]. Because of the very small cross-sections a very limited number of ${ }^{26} \mathrm{Al}$ atoms is produced, which requires both, a high overall efficiency of the measurement and very low background interference.

Another prominent example for applying AMS is the neutron capture cross section of ${ }^{62} \mathrm{Ni}$ producing the radionuclide ${ }^{63} \mathrm{Ni}$. This reaction plays an important role in the control of the flow path of the slow neutron-capture nucleosynthesis process $[27,28]$. Its cross-section was identified as a key value to describe correctly the isotopic pattern around ${ }^{62} \mathrm{Ni}[29,30]$. AMS has also been used to determine the production of ${ }^{44} \mathrm{Ti}$ via the ${ }^{40} \mathrm{Ca}(\alpha, \gamma)$ reaction [19]. Apart from these examples, a lot of additional measurements have been performed or are being performed using AMS. At VERA a measurement program is underway for studying neutron capture reactions for various isotopes, too.

\section{Ca-measurements with VERA}

Another AMS nuclide is ${ }^{41} \mathrm{Ca}$. This radioisotope has a half-life of 104000 years (see, e.g., [31]). The long halflife together with its direct electron-capture decay to the ground state of ${ }^{41} \mathrm{~K}$ makes this nuclide difficult to detect via decay counting. The measurement of this nuclide is of interest in astrophysics for a quantification of the ${ }^{40} \mathrm{Ca}(\mathrm{n}, \gamma)$ cross section at stellar energies, which is underway at different laboratories. In the following some recent developments at VERA for the detection of the long-lived radionuclide ${ }^{41} \mathrm{Ca}$ are presented.

Natural ${ }^{41} \mathrm{Ca} / \mathrm{Ca}$ isotope ratios are of the order of $10^{-14}$ to $10^{-15}$. Such low ratios have been measured at other AMS laboratories using $\mathrm{CaH}_{2}$ samples for AMS. $\mathrm{CaH}_{2}$ needs a very elaborate sample preparation and sample handling. This chemical form was necessary because the stable isobar ${ }^{41} \mathrm{~K}$ does not form stable negative ${ }^{41} \mathrm{KH}_{3}^{-}$ -ions. Thus, using $\mathrm{CaH}_{2}$, effective isobar suppression is achieved and isotope ratios of the order of $10^{-15}$ can be quantified using AMS [32,33]. However, in cases where the ${ }^{41} \mathrm{Ca}$ content is not as low as in natural concentrations, i.e. for isotope ratios of the order of $10^{-13}$ or higher, an alternative chemical form can be used. Calcium fluoride samples, $\mathrm{CaF}_{2}$, have been proven to be a suitable material for such applications with much simpler chemical preparation.

Here we want to present first results on ${ }^{41} \mathrm{Ca}$ detection with a $3 \mathrm{MV}$ tandem accelerator using $\mathrm{CaF}_{2}$. To this end a new isobar discrimination technique, the so-called deltaTOF technique $[34,35]$ has been applied. Note, ${ }^{41} \mathrm{Ca}$ is not a typical nuclide for small accelerators since isobaric interferences at this mass range are usually not sufficiently resolved by standard AMS-techniques having particle energies below $1 \mathrm{MeV} / \mathrm{amu}$. The measurement of $\mathrm{CaF}_{2}$ samples at VERA has become possible by the availability of so-called silicon nitride ( $\mathrm{SiN}$ ) foils, which offer a very homogenous foil thickness. The method applied is the following: mass selected ions pass through sufficiently thick degrader foils and loose energy, depending on their atomic number $Z$. Thus isobars, which have initially the same velocity, have different velocities after the foil which is measured using a high resolution time-of-flight setup. The homogeneity of those SiN-foils yields resolvable energy discrimination above the inherent energy straggling.

We could utilize a series of ${ }^{41} \mathrm{Ca}$ standards, which were kindly supplied by K. Nishiizumi [36] and G. Korschinek. They span a range of isotope ratios from $1.2 \cdot 10^{-10}$ and $5.9 \cdot 10^{-13}$. These standards allow us to optimize our AMSsetup and to investigate the limits of a $3 \mathrm{MV}$ tandem accelerator for quantifying ${ }^{41} \mathrm{Ca}$ using $\mathrm{CaF}_{2}$ samples. 


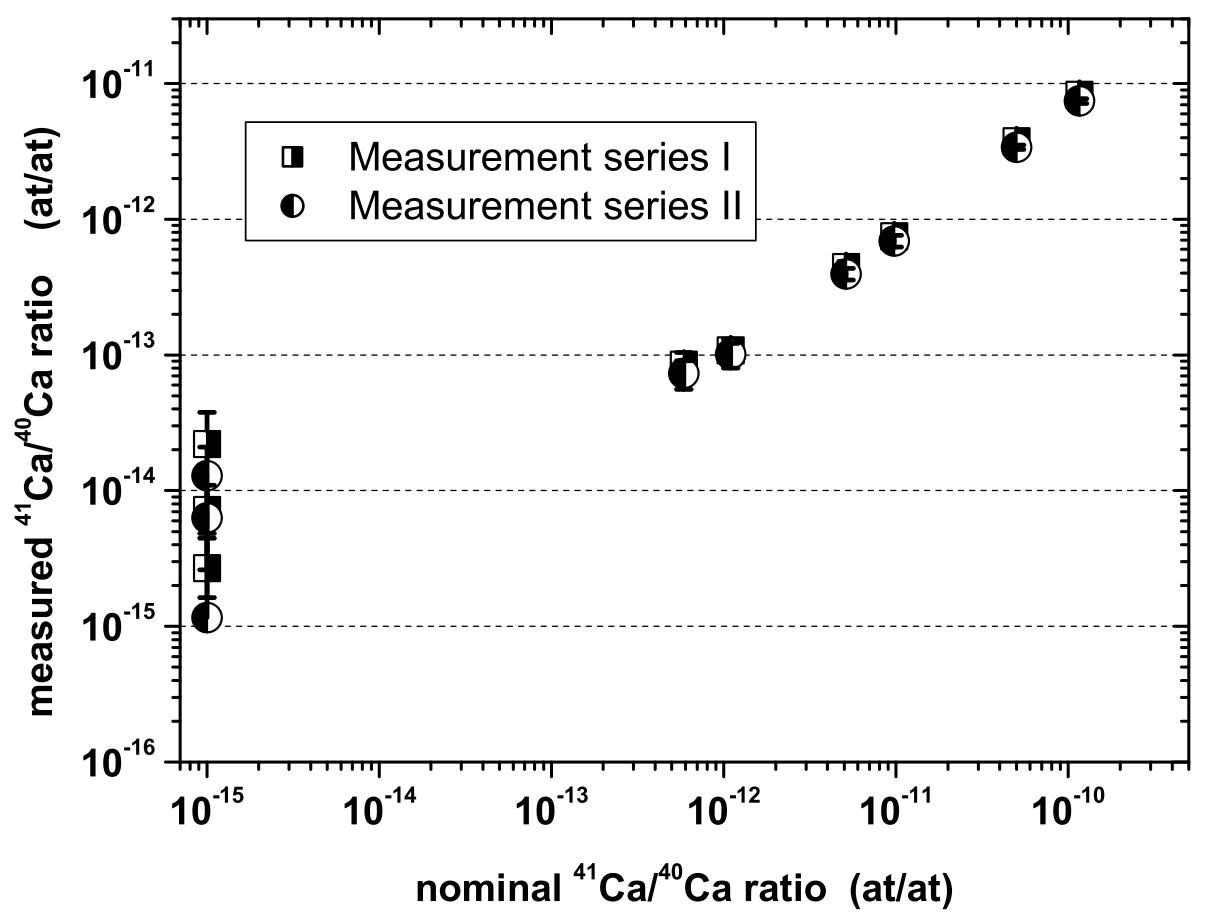

Fig. 2. Measurement of a ${ }^{41} \mathrm{Ca}$-standard dilution series. The nominal ratios represent the quoted ${ }^{41} \mathrm{Ca} /{ }^{40} \mathrm{Ca}$ isotope ratios, the measured ${ }^{41} \mathrm{Ca} /{ }^{40} \mathrm{Ca}$ ratio is the raw uncorrected ratio of the ${ }^{41} \mathrm{Ca}$ count-rate and the ${ }^{41} \mathrm{Ca}$ particle current. Blank samples with expected negligible ${ }^{41} \mathrm{Ca}$ content were assigned to a nominal ratio of $1 \cdot 10^{-15}$.

The measurement procedure applied at VERA is the following: in the cesium sputter source, negatively charged ${ }^{41} \mathrm{CaF}_{3}^{-}$-ions are produced and injected into the tandem accelerator, which is set to a voltage of $3.3 \mathrm{MV}$. After the tandem accelerator, ${ }^{41} \mathrm{Ca}^{4+}$ ions, having energies of about $15 \mathrm{MeV}$, are identified with the detector setup described above. However, the detection efficiency drops with the additional scattering when the beam is passing the thick SiN foils. To this end, the flight path has been reduced from $2.2 \mathrm{~m}$ as used for heavy ion measurements (see fig. 1) to $0.65 \mathrm{~m}$. The time resolution is still sufficient since energyloss straggling is the main contribution to the width of the peaks. Besides the time-of-flight information, utilizing an ionization chamber at the very end of the beam-line, the total residual energy of the incoming ions is measured, too.

Six different Ca-standards have been measured applying this technique. In fig. 2 the measured ${ }^{41} \mathrm{Ca} /{ }^{40} \mathrm{Ca}$ isotope ratios are plotted versus their nominal values. Measured data represent the absolute ratios, uncorrected for background events. The absolute value of these data reflects the beam losses between the position of the current measurement and the particle detection system. Losses are mainly due to the beam scattering in the thick SiN foils. The data should follow a straight line if background interference is of no concern. Blank samples are plotted at the left part of the figure assigning them a nominal ratio of $10^{-15}$. The background value found for such samples during these measurements was about 3 orders of magnitude lower than the value of the highest $\mathrm{Ca}$ standard with a quoted ratio ${ }^{41} \mathrm{Ca} /{ }^{40} \mathrm{Ca}$ of $1.16 \cdot 10^{-10}[36]$, which results in an normalized value of about $1 \cdot 10^{-13}$ for that blank samples. The uncertainty of these blank samples enters into the final values and dominates already the error of the standard material with the (lowest) nominal value of $5.9 \cdot 10^{-13}$ (see error bars). Different symbols in the plot depict the use of SiN degrader foils of different thickness: measurement series I was carried out using $1000 \mathrm{~nm}$ thick SiN foils and measurement series II using a $1650 \mathrm{~nm}$ thick foil. This figure clearly shows that ${ }^{41} \mathrm{Ca} /{ }^{40} \mathrm{Ca}$ isotope ratios of a few $10^{-13}$ can be quantified with this setup. In a later measurement series the K-background showed a somewhat enhanced count rate, which was up to a factor of 20 higher than for the previous measurements. A possible reason for this higher background rate might be the preceding cleaning of the ion source which was combined with the replacement of some source parts. Those new parts are suspicious to K-contamination. A systematic investigation of possible sources for this enhanced background is presently underway.

\section{Summary}

It has been shown - for the first time - that a $3 \mathrm{MV}$ tandem accelerator is capable to measure ${ }^{41} \mathrm{Ca} /{ }^{40} \mathrm{Ca}$ ratios down to a few $10^{-13}$. The reproducibility of those measurements was found to be between 5 to $8 \%$. Applying the deltaTOF technique allows the use of $\mathrm{CaF}_{2}$ samples despite potassium will form negative ions and therewith isobars of ${ }^{41} \mathrm{Ca}$ will be produced, too. For samples with ratios on the order of or below $10^{-12}$ a moderate ${ }^{41} \mathrm{~K}$ 
background count-rate will become desirable because it may dominate the uncertainty of the $\mathrm{Ca}$ data.

Our recent investigations on ${ }^{41} \mathrm{Ca}$ measurements at VERA opens now the possibility for quantitative Ca measurements. As a first project, the determination of the cross section for the ${ }^{40} \mathrm{Ca}(\mathrm{n}, \gamma){ }^{41} \mathrm{Ca}$ reaction at astrophysical interesting neutron energies $(25 \mathrm{keV})$ is in progress.

We thank F. Käppeler and M. Paul for including VERA into the ${ }^{40} \mathrm{Ca}(\mathrm{n}, \gamma)$ measurement program. We gratefully acknowledge K. Nishiizumi and G. Korschinek for leave some Ca standard material for our measurement program.

\section{References}

1. L. Buchmann, M. Comyn, J. Thomson (Editors) Proceedings of the 8th International Symposium on Nuclei in the Cosmos Vancouver, British Columbia, Canada, 19-23 July, 2004, Nucl. Phys. 758, 3-859 (2005).

2. F.-K. Thielemann, D. Argast, F. Brachwitz, J.L. Fisker, C. Fröhlich, R. Hirschi, E. Kolbe, D. Mocelj, T. Rauscher, Nucl. Phys. A 751, 301 (2005).

3. M. Wiescher, Nucl. Phys. A 751, 285 (2005).

4. W. Kutschera, P. Collon, H. Friedmann, R. Golser, P. Hille, A. Priller, W. Rom, P. Steier, S. Tagesen, A. Wallner, E. Wild, G. Winkler, Nucl. Instrum. Methods B $\mathbf{1 2 3}$ 47 (1997).

5. P. Steier, R. Golser, W. Kutschera, A. Priller, C. Vockenhuber, S. Winkler, Nucl. Instrum. Methods B 223-224, 67 (2004).

6. C. Vockenhuber, I. Ahmad, R. Golser, W. Kutschera, V. Liechtenstein, A. Priller, P. Steier, S. Winkler, Int. J. Mass Spectrom. 223, 713 (2003).

7. W. Kutschera, Nucl. Phys. A 752, 645 (2005).

8. W. Kutschera, Int. J. Mass Spectrom. 242, 145 (2005).

9. A. Wallner, R. Golser, W. Kutschera, A. Priller, P. Steier, C. Vockenhuber, H. Vonach, T. Faestermann, K. Knie, G. Korschinek, R.C. Haight et al. (Editors), AIP Conf. Proc. 769, 621 (2005).

10. J. Ellis, B.D. Fields, D.N. Schramm, Astrophys. J. 470, 1227 (1996).

11. B.D. Fields, New Astron. Rev. 48, 119 (2004).

12. K. Knie, G. Korschinek, T. Faestermann, E.A. Dorfi, G. Rugel, A. Wallner, Phys. Rev. Lett. 93, 171103 (2004).

13. B.D. Fields, K.A. Hochmuth, J. Ellis, Astrophys. J. 621, $902(2005)$.

14. C. Vockenhuber, C. Feldstein, M. Paul, N. Trubnikov, M. Bichler, R. Golser, W. Kutschera, A. Priller, P. Steier, S. Winkler, New Astron. Rev. 48, 161 (2004).

15. S. Winkler, I. Ahmad, R. Golser, W. Kutschera, K.A. Orlandini, M. Paul, A. Priller, P. Steier, C. Vockenhuber, New Astron. Rev. 48, 151 (2004).
16. P. Steier, R. Golser, W. Kutschera, V. Liechtenstein, A. Priller, A. Valenta, C. Vockenhuber, Nucl. Instrum. Methods B 188, 283 (2002).

17. E. Hrnecek, P. Steier, A. Wallner, Appl. Radiat. Isotopes 63, $633(2005)$.

18. C. Wallner, T. Faestermann, U. Gerstmann, K. Knie, G. Korschinek, C. Lierse, G. Rugel, New Astron. Rev. 48, 145 (2004).

19. M. Paul, A. Valenta, I. Ahmad, D. Berkovits, C. Bordeanu, S. Ghelberg, Y. Hashimoto, A. Hershkowitz, S. Jiang, T. Nakanishi, K. Sakamoto, Nucl. Phys. A 719, C29 (2003).

20. C. Wallner, T. Faestermann, U. Gerstmann, W. Hillebrandt, K. Knie, G. Korschinek, C. Lierse, C. Pomar, G. Rugel, Nucl. Instrum. Methods B 172, 333 (2000).

21. W. Prantzos, R. Diehl, Phys. Rep. 267, 1 (1996).

22. A. Wallner, S.V. Chuvaev, A.A. Filatenkov, Y. Ikeda, W. Kutschera, G. Mertens, A. Priller, W. Rochow, P. Steier, H. Vonach, Eur. Phys. J. A 17, 285 (2003).

23. A. Wallner, Y. Ikeda, W. Kutschera, A. Priller, P. Steier, H. Vonach, E. Wild, Nucl. Instrum. Methods B 172, 382 (2000).

24. A. Arazi, T. Faestermann, J.O. Fernández Niello, K. Knie, G. Korschinek, M. Poutivtsev, E. Richter, G. Rugel, A. Wallner, submitted to Phys. Rev. C (2006).

25. A. Arazi, T. Faestermann, J.O. Fernández Niello, D. Frischke, K. Knie, G. Korschinek, H.J. Maier, E. Richter, G. Rugel, A. Wallner, Nucl. Instrum. Methods B 223-224, 259 (2004).

26. A. Arazi, T. Faestermann, J.O. Fernández Niello, K. Knie, G. Korschinek, E. Richter, G. Rugel, A. Wallner, New Astron. Rev. 46, 525 (2002).

27. H. Nassar et al., Phys. Rev. Lett. 94, 092504 (2005).

28. A. Tomyo et al., Astrophys. J. 623, L153 (2005).

29. T. Rauscher, K.H. Guber, Phys. Rev. C 66, 028802 (2002); T. Rauscher, K.H. Guber, Phys. Rev. C 71, 059903(E) (2005).

30. M. Paul et al., Nucl. Phys. A 718, 239c (2003); H. Nassar et al., Nucl. Phys. 758, 411 (2005).

31. M. Paul, I. Ahmad, W. Kutschera, Z. Phys. A 340, 249 (1991); W. Kutschera, I. Ahmad, M. Paul, Radiocarbon 34, 436 (1992).

32. W. Kutschera, I. Ahmad, P.J. Billquist, B.G. Glasgola, K. Furer, R.C. Pardo, M. Paul, K.E. Rehm, P.J. Slota jr., R.E. Taylor, J.L. Yntema, Radiocarbon 31, 311 (1989).

33. D. Fink, R. Middleton, J. Klein, P. Sharma, Nucl. Instrum. Methods B 47, 79 (1990); D. Fink, J. Klein, R. Middleton, Nucl. Instrum. Methods B 52, 572 (1990).

34. P. Steier, R. Golser, V. Liechtenstein, W. Kutschera, A. Priller, C. Vockenhuber, A. Wallner, Nucl. Instrum. Methods B 240, 445 (2005).

35. C. Vockenhuber, R. Golser, W. Kutschera, A. Priller, P. Steier, K. Vorderwinkler, A. Wallner, Nucl. Instrum. Methods B 240, 490 (2005).

36. K. Nishiizumi, M.W. Caffee, D.J. DePaolo, Nucl. Instrum. Methods B 172, 399 (2000). 